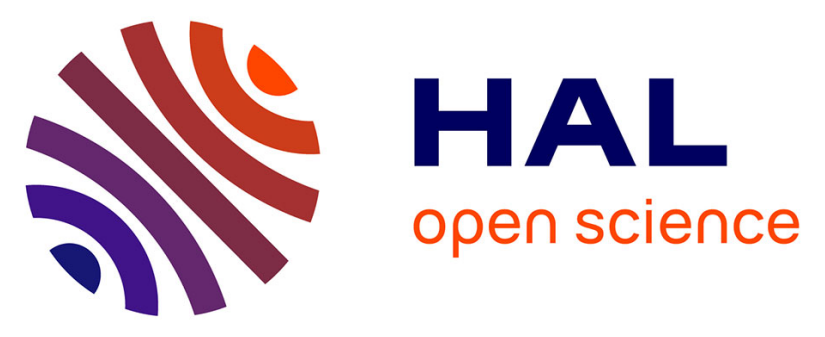

\title{
Strongly emissive plasma-facing material under space-charge limited regime: Application to emissive probes
}

Jordan Cavalier, Nicolas Lemoine, Guillaume Bousselin, Nicolas Plihon, Jordan Ledig

\section{To cite this version:}

Jordan Cavalier, Nicolas Lemoine, Guillaume Bousselin, Nicolas Plihon, Jordan Ledig. Strongly emissive plasma-facing material under space-charge limited regime: Application to emissive probes. Physics of Plasmas, 2017, 24 (1), pp.13506 - 13506. 10.1063/1.4973557 . hal-01576912

\section{HAL Id: hal-01576912 \\ https://hal.univ-lorraine.fr/hal-01576912}

Submitted on 24 Aug 2017

HAL is a multi-disciplinary open access archive for the deposit and dissemination of scientific research documents, whether they are published or not. The documents may come from teaching and research institutions in France or abroad, or from public or private research centers.
L'archive ouverte pluridisciplinaire HAL, est destinée au dépôt et à la diffusion de documents scientifiques de niveau recherche, publiés ou non, émanant des établissements d'enseignement et de recherche français ou étrangers, des laboratoires publics ou privés.

\section{()ㅜㅇㅜ}

Distributed under a Creative Commons Attribution - NoDerivatives| 4.0 International 


\title{
Strongly emissive plasma-facing material under space-charge limited regime: Application to emissive probes
}

\author{
Jordan Cavalier, ${ }^{1,2}$ Nicolas Lemoine, ${ }^{2}$ Guillaume Bousselin, ${ }^{3}$ Nicolas Plihon, ${ }^{3}$ and Jordan Ledig ${ }^{2}$ \\ ${ }^{1}$ Institute of Plasma Physics, AS CR, v.v.i, Za Slovankou 3, Prague 8, Czech Republic \\ ${ }^{2}$ Institut Jean Lamour IJL, Université de Lorraine, \\ UMR 7198 CNRS, Vandouvre-lès-Nancy, France \\ ${ }^{3}$ Univ Lyon, Ens de Lyon, Univ Claude Bernard, \\ CNRS, Laboratoire de Physique, F-69342 Lyon, France
}

A quasi-static theoretical 1D model is developed to describe the sheath structure of a strongly emissive plasma-facing material and is subsequently applied to emissive probes experimental data which are usually supposed to be an efficient tool to directly measure plasma potential fluctuations. The model is derived following the space-charge limited emission current model developed in [S. Takamura, N. Ohno, M.Y. Ye, and T. Kuwabara. Contribution to Plasma Physics, 44(1-3):126-137, 2004.], adding the contribution of secondary emission due to back-diffusion of plasma electrons at the emitting surface. From this theory, current-voltage characteristics of emissive probes are derived. A theoretical relation between the floating potential of an emissive probe and plasma parameters is obtained and a criterion is derived to determine the threshold between thermoemission limited current regime and space-charge limited current regime. In the space-charge limited regime, a first order expansion is then applied to the quasi-static relation to study the effect of plasma fluctuations on emissive probe measurements. Both the mean values and the fluctuations of the floating potential of an emissive probe predicted by the model, as well as the potential value at which the transition between emission current regimes occurs, are compared to three sets of experimental data obtained in two different plasma devices. 


\section{INTRODUCTION}

The study of electron emitting surfaces is of great interest for plasma physics. First, an emissive enough floating conducting surface has a mean electric potential close to that of the surrounding plasma. This has led to the wide use of emissive probes [20,21] in which the floating potential of a strongly emissive material is interpreted as a direct measurement of plasma potential - up to frequencies low compared to the ion plasma frequency. Second, when a surface becomes emissive, emitted electrons modify the electric potential in the sheath ahead, which in turn affect thermal fluxes on the surface. This question has strong implications in the field of magnetic controlled fusion. For instance, the divertor in ITER (that will receive most of the thermal flux from the plasma) is expected to reach temperatures so high that it could be strongly emissive.

A precise understanding of the sheath structure facing emissive material surface is thus required to model incident particles and heat fluxes on the surface. Restricting ourselves to electrostatic probes, this is a recurrent problem for the analysis of emissive probes heated by an external controlled process, often used in low-temperature plasmas to measure the plasma potential and its low-frequency fluctuations [20]. This is also an important issue for reciprocating probes, used to study edge plasma in tokamaks, which are plunged during a very short time into hot plasmas that makes them occasionally self-emissive [2]. In the context of fusion plasmas, a precise estimation of particle and heat transport requires a precise measurement of the phase between plasma density and plasma potential fluctuations, and emissive probes are considered for the measurement of the latest (see the undergoing development of a probe head for TCV and ASDEX UG under the WPMST2 project [1] and a newly developed emissive probe [18]). A precise understanding of the behavior of emissive probe is thus a key issue when addressing turbulent plasma transport, especially in the case of drift wave turbulence.

While it is now widely accepted that the time-averaged value of the floating potential of emissive probes can equal the surrounding plasma potential in cold plasmas [3], it has been recently shown that the fluctuations of the floating potential can differ significantly from the fluctuations of the plasma potential, both in phase and amplitude [4, 5]. Moreover, the experimental investigation reported in [5] demonstrated that the fluctuation level of the floating potential of emissive probes follows a thermoemission limited current model only for low emission currents. Above a critical value of the emission current, fluctuations of the floating potential go clearly off the model curve. This nonmonotonic evolution of the floating potential evolution is suspected to be the signature of a transition to a space-charge limited current regime.

In this article, we develop a theoretical framework to account for the two above observations. More specifically, we derive current-voltage characteristics of emissive probes in the space-charge limited regime, as the classical model for thermoemission limited current regime did not account for experimental measurements described in Ref. [4, 5]. The thermoemission regime exists as long as the potential profile in the sheath is monotonic (see Fig. 1(a)), so that the emission current density reaching the plasma is only limited by the wall emissivity (usually controlled by its temperature). The transition to the space-charge regime is observed when the low energy electrons emitted from the surface would have a too high current density or would not be accelerated enough by the sheath drop, so that their density at the plasma/sheath edge would be too large and, eventually, break plasma quasineutrality, if the potential profile were to be monotonic. Before that occurs, a virtual cathode appears in front of the wall that has a lower potential than the wall potential. It filters out the low energy emitted electrons in such a way that a given current density of passing emitted electrons is associated to a lower density at the plasma/sheath boundary. Note that the potential drop also repels a fraction of incoming electrons. The existence of virtual cathodes was postulated and demonstrated by the pioneering work of Langmuir [12], and then investigated in details by Kemp and Sellen [10]. More recently, detailed measurements of virtual cathode structures caused by secondary electron emission [13] have been reported in low density plasmas. In this article, we extend the work presented in Ref. [23] by adding the effect of secondary electron emission by retrodiffusion of primary electrons. The addition of this new ingredient is motivated by the fact that between 10 and 40 percent of primary electrons reaching a tungsten wall could bounce on it [11]. Recently, even larger values have been measured on CFC component in the plasma edge of a tokamak [8]. This has implication on the mean floating potential value of the probe as well as on its fluctuations as the rebound current would follow plasma fluctuations. The model developed in this work enables to provide predictions for the relations between fluctuations of the floating potential of emissive probes and fluctuations of the plasma potential in the space-charge regime. It is then compared to experimental results.

The article is organized as follows. In section II, a model for the space-charge limited emission current regime is derived, following the calculation given in Ref. [23] and adding retrodiffusion of primary electrons. In this section, we also derive an analytical expression of the potential drop between the wall and the virtual cathode potentials. We then show how to obtain $I-V$ characteristics from the model and discuss the implications of the rebound electrons on the sheath characteristics, and hence floating potentials, virtual cathode potential drop or particles fluxes at the emissive surface. In sections III and IV, this model is compared to experimental results. In section III, the onset temperature at which the emissive wall enters the space-charge limited regime is derived and compared to experimental data from 
emissive probes. In section IV, we compare fluctuations from the space-charge model developed in this article and experimental data. In section $\mathrm{V}$, results are discussed and we come to a conclusion.

\section{CURRENT LIMITED BY SPACE-CHARGE REGIME}

This section is devoted to the derivation of a 1D model of a plasma sheath in front of an emissive wall when the emission current is large enough to be limited by space-charge effect. In short, when the current density of electron emitted from the wall is low enough, the potential profile in the sheath is monotonic; this regime is usually referred to as the thermoemission current limited regime since the amount of emitted electrons reaching the plasma is controlled by the emissivity of the wall (which is usually set by the temperature for a given type of emissive material such as tungsten or $\left.\mathrm{LaB}_{6}\right)$. On the other hand, when the current density of electron emitted from the wall is large enough, the potential profile in the sheath is non monotonic. The minimum value of the potential profile is no more reached at the wall, but within the sheath, giving rise to a so-called virtual cathode (in other words, this is obtained when the equilibrium relations require a negative electric field at the wall). The existence of this virtual cathode leads to a reflection of part of the emitted electrons back to the wall; this regimes is usually referred to as the space-charge current limited regime since the amount of emitted electrons reaching the plasma is mainly controlled by the potential drop between the wall potential and the virtual cathode. Fig. 1 shows a schematic view of the sheath structure and associated particles fluxes in both regimes, in which the minimum of the sheath potential is located at $x=0$. Fig. 1 (a) displays the thermoemission current limited regime: the potential profile is monotonic and the electric is oriented towards the wall at the wall location. Fig. 1(b) displays the space-charge current limited regime: the potential profile is non-monotonic and the electric is oriented outwards of the wall at the wall location. The transition between both regimes is observed for a zero electric field at the wall. In the remaining of this sections, one will restrict oneself to the space-charge current limited regime and derive a model for the sheath including rebound electrons, which are shown to strongly influence the sheath characteristics.

\section{A. Hypothesis of the model and outline}

Most of the hypothesis and conventions necessary for the derivation of this model are summarized in Fig. 1(b) which displays typical spatial evolutions of the sheath potential. A hot emissive wall at a wall temperature $T_{w}$ and potential $\varphi_{w}$ is facing a plasma, supposed to be at infinity, of density $n_{0}$, whose plasma potential is $\varphi_{p}$. The distribution function of electrons from the plasma is assumed to be a Maxwellian with electron plasma temperature $T_{e}^{p}$ and the ions, of mass $m_{i}$, are considered cold. A Maxwellian distribution function is also assumed for the electrons emitted by the hot emissive wall, with a temperature $T_{e}^{e}=T_{e}^{p} / C$. We assume that the plasma is hot enough so that $T_{e}^{p}$ is greater than $T_{e}^{e}$, and hence the constant $C$ is greater than one. Note also that $T_{e}^{e}$ could differ from $T_{w}$. In the remaining, the superscript $p$ stands for plasma or primary electrons, and the superscript $e$ stands for emitted electrons at the wall, while Takamura and co-workers used a superscript $s$ standing for secondary electrons; our choice was motivated to avoid confusion with true secondary emission. The sheath is assumed to be collisionless, implying energy and flux conservation. The electron mass will be denoted as $m_{e}$, and the elementary charge as $e$. Normalized potentials $\Phi$ are defined as $\Phi=\left(\varphi-\varphi_{p}\right) / T_{e}^{p}$; dimensional potentials and temperatures are given in volts and in electron-volts, respectively.

For simplicity, the problem is assumed to be one dimensional and static. The hot emitting surface emits a sufficient amount of electrons so that a drop of potential occurs in front of the wall. The space potential $\Phi(x)$ is equal to $\Phi_{w}$ at the wall location $x=x_{w} \geq 0$ and reaches a minimum $\Phi_{v c}$ at the virtual cathode location $x=0$. The sheath entrance or plasma limit is at $x \rightarrow-\infty$. Following the notations of reference [23] (see also Fig. 1 (b)), the sheath region at $x<0$ is labeled $\alpha$ (i.e. between the plasma and the virtual cathode), while the sheath region at $x>0$ is labeled $\beta$ (i.e. between the virtual cathode and the wall).

We follow the lead of Takamura and co-workers [23] and add to the calculation a new population of electrons: the primary electrons that have rebounded elastically on the material as their contribution could be non-negligible. As a novelty, we also derive a model for the potential of a floating material by establishing numerically the dependence of the virtual cathode potential with the different plasma parameters. In addition, we give a method for calculating the emission electron temperature at which occurs the transition between current limited by thermoelectron emission and the space-charge limited regime.

For the sake of clarity, and before detailing the derivation, we first provide a brief outline of the following subsections which establish a relation between $\Phi_{w}$ and $\Phi_{v c}$, as well as a framework for numerical integration of $I$ - $V$ characteristic of emissive probes in the space-charged limited regime. From the dynamics of charged particle populations in regions $\alpha$ and $\beta$, we seek expressions of the current densities associated to each of these populations in subsection II B. In 

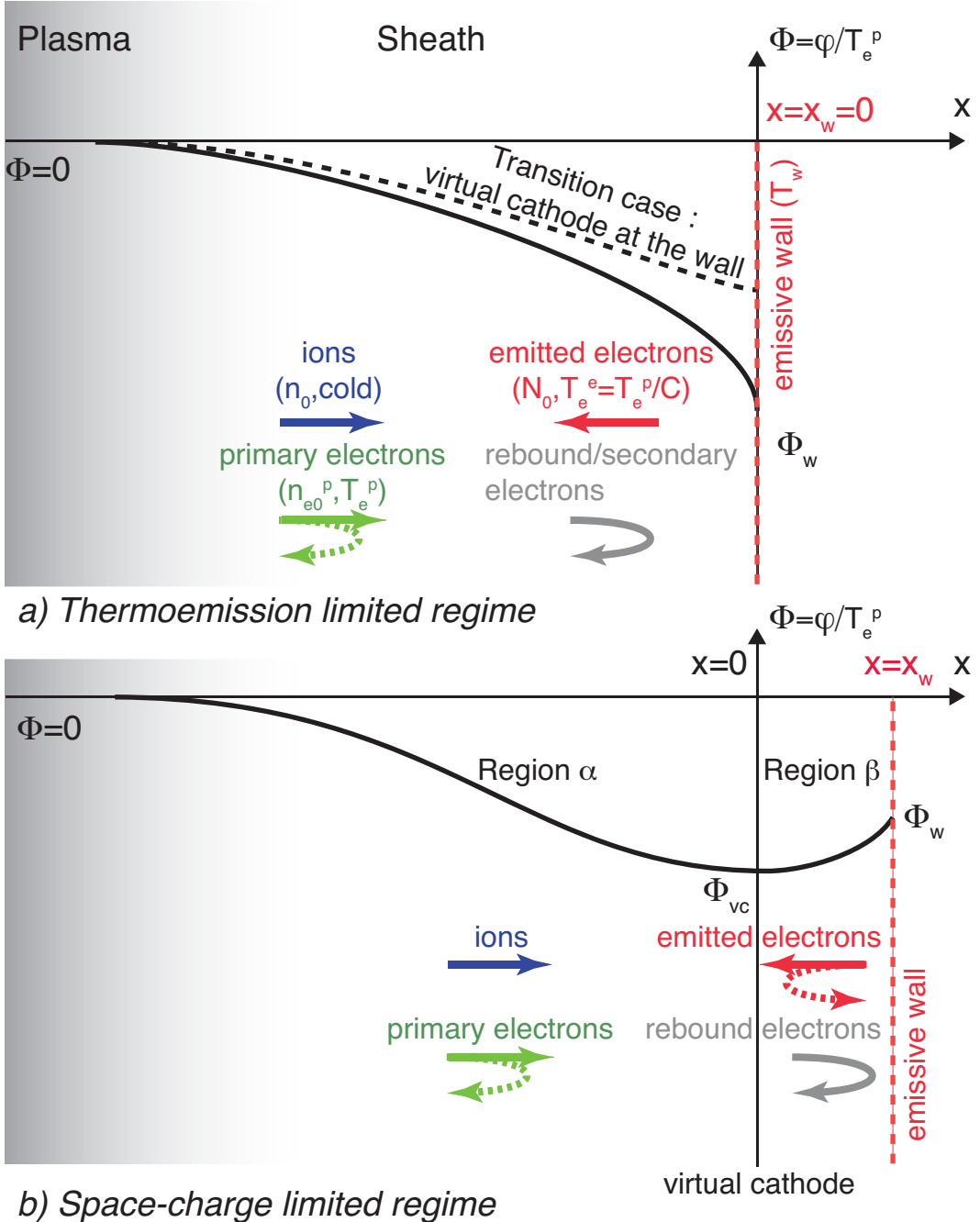

FIG. 1. Schematic of 1-D static sheath under (a) thermoemission limited regime (b) space-charge limited regime. See text for details.

subsection II C, these current densities are then expressed as a function of $\Phi_{v c}, \Phi_{w}, M, \gamma$, where $M$ is the Mach number and $\gamma$ is the ratio of passing emitted electrons current density over the passing plasma electron current density. In subsection IID, a modified Bohm criterion, derived from Poisson's equation in region $\alpha$, enables to express $M$ as a function of $\gamma$ and $\Phi_{v c}$, and a relation between $\gamma$ and $\Phi_{v c}$ is also obtained from the condition of zero electric field at the virtual cathode (or equivalently minimum of potential). Finally, in subsection II E, matching the current densities computed for each population at the virtual cathode leads to an explicit relation between $\Phi_{v c}$ and $\Phi_{w}$ that leads to a theoretical expression of the wall floating potential or to numerical integration of $I-V$ characteristic of emissive probes under space-charge regime.

\section{B. Densities in region $\alpha$ and current densities of passing particles}

In this subsection, particle densities for each population are expressed in the region $\alpha$. The current densities of passing particles, those able to override the potential barrier created by the virtual cathode, are then derived.

Cold plasma ions. Let us first deal with the dynamics of ions. Assuming that cold ions of mass $m_{i}$ are accelerated from the plasma in the sheath potential with no collisions, the ion density $n_{i}(x)$ and ion velocity $v_{i}(x)$ are derived from flux conservation $d\left(n_{i} v_{i}\right) / d x=0$ and energy conservation $m_{i} v_{i}(x)^{2}+2 e T_{e}^{p} \Phi(x)=$ Cte, as: 


$$
v_{i}(x)=\sqrt{V_{0}^{2}-\frac{2 e T_{e}^{p} \Phi(x)}{m_{i}}} \text { and } n_{i}(x)=n_{0}\left(1-\frac{2 \Phi(x)}{M^{2}}\right)^{-1 / 2}
$$

where $n_{0}$ is the ion density in the plasma (at $\left.x \rightarrow-\infty\right), V_{0}$ is the ion velocity at the sheath entrance, and the Mach number at the sheath entrance $M$ is defined as $M=V_{0} / c_{s}$ with the sound speed $c_{s}=\sqrt{e T_{e}^{p} / m_{i} k_{B}}, k_{B}$ being the Boltzmann constant. Since ions are collisionless, the ion current density $j_{i}(x)$ is constant in the region $\alpha$ and reads

$$
j_{i}(x)=j_{i s}=e n_{0} M c_{s}
$$

where $j_{i s}$ is the ion saturation current.

Electrons emitted by the wall. In the space-charge limited regime, only part of the electrons emitted by the wall, those having a sufficiently high velocity to override the virtual cathode potential barrier $\left(\Phi_{v c}-\Phi_{w}\right)$, reach the $\alpha$ region. The calculation, detailed in the work by Takamura and coworkers [23], is only briefly recalled here. Assuming energy and flux conservation and a Maxwellian distribution function $f^{e}\left(v_{e}\right)$ of emitted electrons at the wall location $x=x_{w}$, the density of passing emitted electrons reads:

$$
n_{e, \text { pass }}^{e}(x)=\frac{N_{0}}{2} \operatorname{erfc}\left(\sqrt{C\left(\Phi(x)-\Phi_{v c}\right)}\right) \exp \left(C\left(\Phi(x)-\Phi_{w}\right)\right)
$$

where $N_{0}$ is the emitted electron density at the wall and erfc $(y)$ the complementary error function defined as $\sqrt{\pi /(4 b)} \operatorname{erfc}(a \sqrt{b})=\int_{a}^{+\infty} e^{-b x^{2}} d x$. It is worth emphasizing that the above expression for the density of emitted electrons $n_{e, \text { pass }}^{e}$ is the total density of emitted electrons in region $\alpha$, while it only represents part of the emitted electrons density in region $\beta$. The mean velocity of passing electrons is then computed as:

$$
\left\langle v_{e}^{e}\right\rangle_{\mathrm{pass}}=-\frac{\int_{-\infty}^{-v_{\text {min }}^{e}} v_{e} f^{e}\left(v_{e}\right) d v_{e}}{\int_{-\infty}^{-v_{m i n}^{e}} f^{e}\left(v_{e}\right) d v_{e}}=-\sqrt{\frac{2 T_{e}^{e}}{\pi m_{e}}} \frac{\exp \left(-C\left(\Phi(x)-\Phi_{v c}\right)\right)}{\operatorname{erfc}\left(\sqrt{C\left(\Phi(x)-\Phi_{v c}\right)}\right)}
$$

where $v_{\text {min }}^{e}=\sqrt{e T_{e}^{p}\left(\Phi_{w}-\Phi_{v c}\right) / m_{e}}$ is the minimum velocity necessary for an electron emitted at the surface to override the virtual cathode potential barrier. The current density of passing emitted electrons reads

$$
j_{e, \mathrm{pass}}^{e}=e n_{e, \mathrm{pass}}^{e}\left\langle v_{e}^{e}\right\rangle_{\mathrm{pass}}=\frac{1}{2} e N_{0} \sqrt{\frac{2 e T_{e}^{e}}{\pi k_{B} m_{e}}} \exp \left(C\left(\Phi_{v c}-\Phi_{w}\right)\right)
$$

It is this emission current that contributes to the probe current as the non-passing electrons are counted once positively when leaving the wall and once negatively when returning, resulting in a zero net current. This current is also called the space-charged limited current.

Primary plasma electrons. In the region $\alpha$ between the plasma and the virtual cathode, the primary electrons are in equilibrium under the action of an electrostatic field and therefore their density follows the Boltzmann law $n_{e}^{p}(x)=n_{e 0}^{p} \exp (\Phi(x))$, where $n_{e 0}^{p}$ is the primary electron density at $x \rightarrow-\infty$. Note that $n_{e 0}^{p} \neq n_{0}$ as some electrons coming from the emissive wall (either emitted or rebound electrons) are also present in the plasma and are to be accounted for quasi-neutrality. The density $n_{e, \text { pass }}^{p}$ and the density current $j_{e, \text { pass }}^{p}$ of passing primary electrons are also estimated similarly to the derivation of Eq. 3, as

$$
n_{e, \text { pass }}^{p}(x)=\frac{n_{e 0}^{p}}{2} \operatorname{erfc}\left(\sqrt{\Phi(x)-\Phi_{v c}}\right) \exp (\Phi(x)) \quad \text { and } \quad j_{e, \text { pass }}^{p}=\frac{1}{2} e n_{e 0}^{p} \sqrt{\frac{2 e T_{e}^{p}}{\pi k_{B} m_{e}}} \exp \left(\Phi_{v c}\right) .
$$

Rebound electrons. We now add a new ingredient that was not incorporated in the work by Takamura and coworkers [23] by considering the part of primary electrons that elastically collides with the wall and go backward. The contribution of such electrons to the total current can be non-negligible as between 10 and 40 percent of primary electrons reaching a tungsten wall bounce on it [11], and up to $80 \%$ at low plasma temperature according to [8] for CFC wall in tokamaks. Moreover, as we consider collisions to be elastic, all primary electrons bouncing on the wall 
have a sufficiently high kinetic energy to override the potential barrier again. Thus the rebound electron density at the wall location $x_{w}$ is defined as $n_{e}^{r}\left(x_{w}\right)=-\gamma_{r} n_{e, \text { pass }}^{p}\left(x_{w}\right)$ with $\gamma_{r}$ the rebound coefficient believed to be between 0.1 and 0.4 as previously stated. Assuming elastic collisions and flux conservation, the current density of rebound electrons is defined as $j_{e}^{r}=-\gamma_{r} j_{e, \text { pass }}^{p}$, and this equality holds everywhere, leading to

$$
j_{e}^{r}=-\frac{\gamma_{r}}{2} e n_{e 0}^{p} \sqrt{\frac{2 e T_{e}^{p}}{\pi k_{B} m_{e}}} \exp \left(\Phi_{v c}\right)
$$

True secondary emission. In this model, the contribution of true secondary electrons is not taken into account. The reason is the following. Secondary electrons are created by collisions with the wall either of passing primary electrons either of returning emitted electrons not able to override the potential barrier. Secondary electrons that are created from passing primary electrons $j_{e, \text { pass }}^{p}$ lead to a very small current at the wall compared to the emitted current (which will be verified a posteriori in section IIE), and thus those passing secondary electrons are a small fraction of the total passing electrons. Very few secondary electrons are created by low energy emitted electrons that bounce in the virtual cathode and come back to the wall as their temperature is low ( $T_{e}^{e} \sim 0.3 \mathrm{eV}$ maximum) leading to a secondary emission coefficient close to zero [11]. Thus, true secondary emission is to be taken into account only in regimes where emission current is temperature limited.

\section{Determination of edge densities}

Before using the different densities in Poisson's equation, one can notice that two constants are to be determined: the density of emitted electrons at the surface $N_{0}$ and the density of primary electrons at the plasma/sheath boundary $n_{e 0}$. Quasineutrality at the plasma/sheath edge leads to the relation

$$
n_{0}=n_{e 0}^{p}+n_{e, \text { pass }}^{e}(-\infty)+n_{e}^{r}(-\infty)
$$

where we recall that $n_{0}$ is the plasma ion density. It is useful to define the ratio $\gamma$ as the ratio of the passing emitted electron current density over the passing plasma electron current density as $\gamma=-j_{e, \text { pass }}^{e} / j_{e, \text { pass }}^{p}$. This $\gamma$ coefficient is similar to the true secondary $\gamma_{s}$ or rebound $\gamma_{r}$ emission and may be understood as an effective coefficient linking the emitted electron current density reaching the plasma with the plasma electron current density reaching the wall in the presence of a virtual cathode. Quasineutrality leads to the following relations

$$
\left\{\begin{array}{l}
N_{0}=\gamma n_{e 0}^{p} C^{-1 / 2} \exp \left(\Phi_{v c}+C\left(\Phi_{w}-\Phi_{v c}\right)\right) \\
n_{e 0}^{p}=\frac{n_{0}}{1+A G+A_{r} G \gamma_{r} / \gamma}
\end{array}\right.
$$

with

$$
\left\{\begin{array}{rrr}
A & =\sqrt{-\pi C \Phi_{v c}} \operatorname{erfc}\left(\sqrt{-C \Phi_{v c}}\right) \exp \left(-C \Phi_{v c}\right) \\
G & = & \frac{\gamma}{2}\left(-\pi \Phi_{v c}\right)^{-1 / 2} \exp \left(\Phi_{v c}\right) \\
A_{r} & = & \sqrt{-\pi \Phi_{v c}} \operatorname{erfc}\left(\sqrt{-\Phi_{v c}}\right) \exp \left(-\Phi_{v c}\right)
\end{array}\right.
$$

\section{Bohm criterion}

The previous analysis shows that there are still four independent parameters for estimating the different currents: the Mach number $M$, the ratio $\gamma$, the virtual cathode potential $\Phi_{v c}$ and the wall potential $\Phi_{w}$ such that the total density current flowing through the wall can be expressed as $j_{t o t}=f\left(M, \gamma, \Phi_{v c}, \Phi_{w}\right)$. In this section, a modified Bohm criteria, classically established by integration of the Poisson's equation, settles $M$ as a function of $\Phi_{v c}$ and $G$ - or equivalently as a function of $\Phi_{v c}$ and $\gamma$ from Eq. 10. Moreover, using the fact that the virtual cathode is a local minimum of the potential (equivalently that the electric field is zero at the virtual cathode), leads to express $\gamma$ as a function of $\Phi_{v c}$. Finally the total current density may be expressed as a function of $\Phi_{w}$ and $\Phi_{v c}$, allowing for the computation of the floating potential of the hot wall or for the computation of the $I-V$ characteristic of emissive probes. 
Introducing $\xi$ as the space variable normalized to the Debye length $\lambda_{D}=\sqrt{\epsilon_{0} e T_{e}^{p} /\left(n_{0} k_{B}\right)}$ with $\epsilon_{0}$ the vacuum permittivity, Poisson's equation in region $\alpha$ reads:

$$
\begin{aligned}
\frac{d^{2} \Phi(\xi)}{d \xi^{2}}= & -\frac{1}{\sqrt{1-\frac{2 \Phi(\xi)}{M^{2}}}}+\frac{1}{1+A G+A_{r} G \gamma_{r} / \gamma}\left[e^{\Phi(\xi)}\left(1+\frac{\gamma_{r}}{2} \operatorname{erfc}\left(\sqrt{\Phi(\xi)-\Phi_{v c}}\right)\right)\right. \\
& \left.+G \sqrt{-\pi \Phi_{v c} C} e^{C\left(\Phi(\xi)-\Phi_{v c}\right)} \operatorname{erfc}\left(\sqrt{C\left(\Phi(\xi)-\Phi_{v c}\right)}\right)\right]
\end{aligned}
$$

This equation can be integrated between $-\infty$ and $\xi$ by multiplying each side by $d \Phi / d \xi$ and employing zero electric field at the plasma/sheath edge as boundary conditions. As the left hand side $(d \Phi / d \xi)^{2}$ is always positive, a solution of this equation only exists if the right hand side is positive as well. This implies a condition on the Mach number $M$ and so on the ion velocity at the entrance of the sheath. Such a condition can be found by making a second order Taylor expansion of the right hand side near the sheath edge $x \rightarrow-\infty$, where $\Phi$ and $d \Phi / d \xi$ tend to zero. We then find a modified Bohm condition for a stable sheath formation which is

$$
M^{2} \geq \frac{1+A G+A_{r} G \gamma_{r} / \gamma}{1+C(A-1) G+G^{2}\left(A_{r}-1\right) \gamma_{r}^{2} / \gamma^{2}}
$$

This criterion is similar to the one obtained in Ref. [23], with additional terms accounting for rebound electrons. Using the fact that the electric field is zero at the virtual cathode (i.e. $d \Phi / d \xi\left(\Phi=\Phi_{v c}\right)=0$ ) and using the marginal condition for the Bohm criterion (i.e. equality condition in Eq. 12) a fourth order polynomial expression in $\gamma$ (through $G)$ is obtained as:

$$
\left(1+C(A-1) G+G^{2}\left(A_{r}-1\right) \gamma_{r}^{2} / \gamma^{2}\right) \times\left(\beta_{0}+\beta_{1} G+\beta_{2} G^{2}+\beta_{3} G^{3}\right)=0
$$

where $\beta_{0}, \beta_{1}, \beta_{2}$ and $\beta_{3}$ are coefficients that are not specified for conciseness and that depend on $A$ and $C$ and thus only on $\Phi_{v c}$ and on the plasma parameters. Solving this polynomial expression allows to express $\gamma$ as a function of $\Phi_{v c}$, so that both $\gamma$ and $M$ are only functions of $\Phi_{v c}$. Roots of $1+C(A-1) G+G^{2}\left(A_{r}-1\right) \gamma_{r}^{2} / \gamma^{2}$ have no physical meaning because it corresponds to $M \rightarrow+\infty$ (Eq. 12). The right-hand part of Eq. 13 can be solved by the Cardan method. It has a positive discriminant and thus three real solutions, from which only one satisfies $G>0$ (see Eq. 10).

\section{E. Relation between $\Phi_{w}$ and $\Phi_{v c}$}

Plugging $N_{0}$ from Eq. 9 in Eq. 5 allows to rewritte the current density of passing emitted electrons as

$$
j_{e, \mathrm{pass}}^{e}=j_{i s}\left[\frac{G}{1+A G+A_{r} G \gamma_{r} / \gamma} \sqrt{-2 \frac{m_{i}}{m_{e}} \Phi_{v c}}\right]
$$

This current density may also be expressed using the Richardson-Dushmann's formula. The emitted current density at the wall $x=x_{w}$ is given by

$$
j_{e, \text { wall }}=\frac{A_{0}}{2} T_{w}^{2} \exp \left(-\frac{\varphi_{\text {work }}}{T_{w}}\right)
$$

where $A_{0}$ the Dushman's constant and $\varphi_{\text {work }}$ the so-called work function.

Accounting for the drop of potential in front of the wall, the current density of passing emitted electrons at the virtual cathode is

$$
j_{e, \text { pass }}^{e}=\frac{A_{0} T_{w}^{2}}{2} \exp \left(-C^{\prime} \Phi_{\mathrm{work}}\right) \exp \left(C\left(\Phi_{v c}-\Phi_{w}\right)\right)
$$

These two expressions enable to relate $\Phi_{w}$ and $\Phi_{v c}$ :

$$
\Phi_{w}=\Phi_{v c}-\Phi_{\text {work }} \frac{C^{\prime}}{C}+\frac{1}{C} \ln \left[\frac{T_{w}^{2} A_{0}}{j_{i s}} \sqrt{-\frac{m_{e}}{8 \Phi_{v c} m_{i}}} \frac{1+A G+A_{r} G \gamma_{r} / \gamma}{G}\right]
$$


where we have introduced $C^{\prime}=T_{e}^{p} / T_{w}$. The influence of rebound on the virtual potential drop $\Phi_{w}-\Phi_{v c}$ is discussed further in next subsection, but Eq. 17 clearly shows that $\Phi_{w}-\Phi_{v c}$ increases with $\gamma_{r}$ and hence with the amount of rebound electrons.

\section{F. Virtual cathode potential drop in front of the probe}

It is now instructive to examine how the virtual cathode potential drop $\Phi_{w}-\Phi_{v c}$ evolves with the values of the plasma parameters and the bias of the wall $\Phi_{w}$. Note that an analytical expression for this potential drop cannot be straightforwardly deduced from Eq. 17, since $A, A_{r}$ and $G$ depend on $\Phi_{v c}$. In order to compute this potential drop, a priori $\Phi_{v c}$ values are set and corresponding $\Phi_{w}$ values are calculated from Eq. 17. Figure 2(a) displays the evolution of the potential drop $\Phi_{w}-\Phi_{v c}$ as a function of the bias $\Phi_{w}$ for different values of the plasma parameters (we recall here that potentials are normalized to the plasma electron temperature $T_{e}^{p}$ ).

A first observation is that the potential drop increases with $\Phi_{w}$; in other words the potential well at the virtual cathode becomes shallower when the probe approaches the plasma potential. This evolution is coherent with the qualitative explanation for the formation of the virtual cathode, given in the introduction and extended in the remaining of this subsection. As $\Phi_{w}$ increases, all other parameters being constant, the low temperature emitted electrons are less accelerated in the sheath potential drop. If the virtual cathode potential drop were to stay constant, the flux of passing emitted electrons would stay constant (as we assume flux conservation). As their velocity at the plasma/sheath boundary would be smaller, their density at that location would increase. To maintain electroneutrality at the plasma sheath edge, a higher virtual cathode drop is necessary to filter out the slowest electrons, so that emitted electrons reaching the plasma have a higher mean velocity. This results in a lower flux and a lower density of emitted electrons at the plasma/sheath boundary. A second observation is that when the probe is significantly emissive and when its potential is close to the plasma potential, the potential drop is a non-negligeable fraction of the plasma electron temperature. Thus, as the temperature of emitted electrons is usually much lower than $T_{e}^{p}$, only a small fraction of the emitted electrons can override the potential barrier (the queue of the velocity distribution). It is thus clear that the emitted electron current at the wall is much larger than the passing emitted electron current, and thus than the passing plasma electron current, which justifies the assumption made at the last paragraph of section II B namely to neglect true secondary emission.

Let us now investigate how the potential drop $\Phi_{w}-\Phi_{v c}$ evolves with the plasma parameters (see Fig. 2 (a)). The plasma density is observed to be a leading parameter: the potential drop increases with decreasing plasma density. This can also be qualitatively understood from the fact that the virtual cathode shields the plasma from a flux of too slow (cold) emitted electrons. For a given emitted electron flux at the wall ( $T_{w}$ is fixed) and for a given wall potential, the emitted electron density at the plasma/sheath boundary is set by the virtual cathode potential drop. Decreasing the electron plasma density, all other parameters being kept constant, requires a larger virtual cathode potential drop to filter out more slow electrons and to reduce the emitted electron density at the plasma/sheath boundary to ensure quasineutrality. This evolution is observed in Fig. 2(a): the virtual cathode potential drop increases by more than $50 \%$ when the plasma density decreases from $10^{18} \mathrm{~m}^{-3}$ (solid black curve) to $10^{17} \mathrm{~m}^{-3}$ (red squares). On the other hand, the plasma electron temperature has a very weak influence on the virtual cathode potential drop $\Phi_{w}-\Phi_{v c}$ : as potentials are normalized to $T_{e}^{p}$, the ratio between the $5 \mathrm{eV}$ (full solid black curve) and $2.5 \mathrm{eV}$ (blue bullets) data, namely $2.11 \pm 0.07$, is weakly significant. We can conclude from this that the virtual cathode potential drop strongly depends on the electron plasma density but not on the primary electron current density, in agreement with the qualitative explanation given above.

Note that for a lower value of the wall temperature (green crosses), the computation according to the space-charge limited regime predicts a negative value for the potential drop; this indeed means that the current is no more limited by space-charge effect but simply by thermoemission.

Let us now focus on the influence of $\gamma_{r}$ on the virtual cathode potential drop that forms in front of the emissive wall. Fig. 2(b) displays the evolution of $\Phi_{w}-\Phi_{v c}$ as a function of $\Phi_{w}$ for the reference plasma parameters and three values of $\gamma_{r}$. Clearly, rebound electrons have no influence when the wall potential is sufficiently negative as most of primary electrons have not enough energy to reach the wall. However, when the wall potential approaches the plasma potential, the virtual cathode potential drop increases with $\gamma_{r}$ and, near the plasma potential, increasing $\gamma_{r}$ from 0 to 0.8 leads to an increase by $10 \%$ of $T_{e}^{p}$ of the virtual cathode potential drop $\Phi_{w}-\Phi_{v c}$ for the reference conditions. Since $T_{e}^{p}$ is usually larger than $T_{e}^{e}$, it means that the fraction of emitted electrons able to override the potential barrier drops significantly, those emitted electron being replaced by primary electrons that rebound on the wall. It is of importance when considering a floating emissive probe because a non negligeable part of current leaving the probe would be replaced by a current fluctuating as the plasma current (see Fig 3 for $\gamma_{r}=0.3$ near the floating potential). This has also implication on thermal flux that would reach the wall as less plasma electrons are able to override the potential barrier and could be an important effect in the divertor of tokamaks, for instance. 

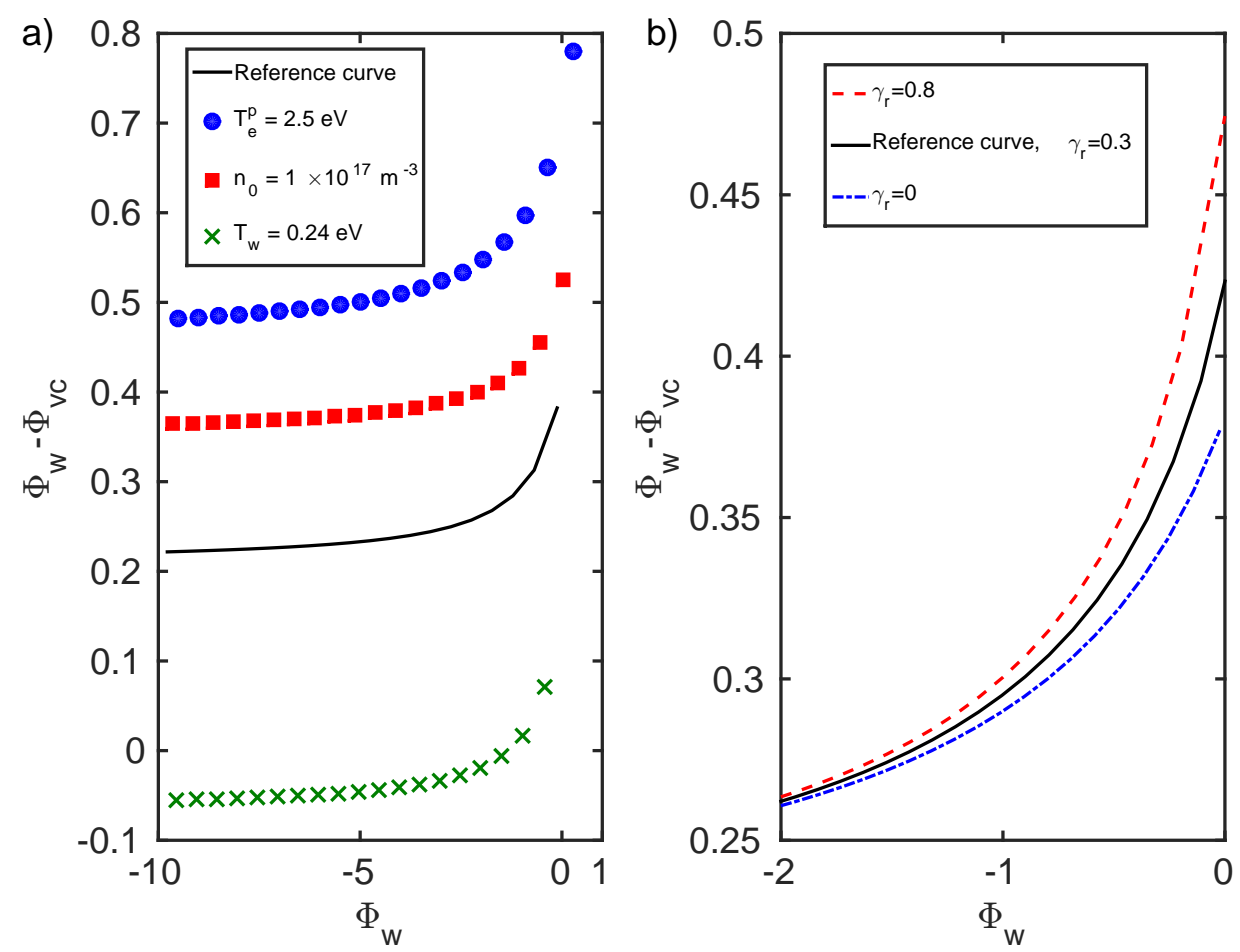

FIG. 2. Evolution of the normalized potential drop $\Phi_{w}-\Phi_{v c}$ as a function of the normalized bias $\Phi_{w}$. The solid black lines in a) and b) correspond to an Argon plasma with $n_{0}=1 \times 10^{18} \mathrm{~m}^{-3}, T_{e}^{e}=T_{w}=0.31 \mathrm{eV}, T_{e}^{p}=5 \mathrm{eV}, \gamma_{r}=0.3, \varphi_{\text {work }}=4.5$ $\mathrm{eV}$ and $A_{0}=1.2 \times 10^{6} \mathrm{Am}^{-2} \mathrm{~K}^{-2}$ (tungsten surface). The other curves are plotted for the same parameters except for the one specified in the legend.

\section{G. $\quad I-V$ characteristics in space-charge regime}

From the previous computation, the total $I$ - $V$ characteristic of an emissive probe in the space-charge limited regime can also be computed. Once the set of parameters $\left(\Phi_{v c}, \Phi_{w}\right)$ is known, the total current $j_{t o t}\left(\Phi_{w}\right)=j_{i}+j_{e, \text { pass }}^{p}+$ $j_{e, \text { pass }}^{e}+j_{e}^{r}$ flowing through an emissive wall biased at the associated potential $\Phi_{w}$ is then computed from Eqs. 2, 5, 6 and 7. An example of two $I-V$ characteristics in the space-charge limited regime are given in Fig. 3 (solid black line for $\gamma_{r}=0.3$ and dashed purple one for $\gamma_{r}=0$ ). Note that for the case $\gamma_{r}=0.3$ and in the vicinity of the floating potential $\left(\Phi_{w} \sim-0.5\right)$, the rebound electron current reaching the probe is a significant part of the total current, on the order of the passing electron emission current. It shows the importance of this electron population when dealing with emissive probes under space-charge effect. One benefit of this computation could be to experimentally measure the coefficient of rebound electrons $\gamma_{r}$. Since the $I-V$ characteristic does not depend on the true secondary emission $\gamma_{s}$ in the space-charge limited regime, a fitting procedure of experimental characteristics would provide an estimate of $\gamma_{r}$, providing that the temperatures of the emissive probe $\left(T_{w}\right)$ and of the emitted electrons $\left(T_{e}^{e}\right)$ are known.

\section{H. Virtual cathode potential for a floating hot surface under space-charge regime}

When the wall is at the floating potential, $\Phi_{w}=\Phi_{f l}$, there is no net current flowing through the wall, $j_{\text {tot }}\left(\Phi_{f l}\right)=0$. For this peculiar condition, $\gamma=-j_{e, \text { pass }}^{e} / j_{e, \text { pass }}^{p}$ may be expressed as a function of the plasma and material parameters $\left(C, \gamma_{r}\right.$ and $\left.m_{i} / m_{e}\right)$ :

$$
\gamma\left(\Phi_{f l}\right)=1-\gamma_{r}-\sqrt{\frac{2 \pi m_{e}}{m_{i}}} M\left(1+A G+A_{r} G \gamma_{r} / \gamma\right) \exp \left(-\Phi_{v c}\right)
$$

In that case, the associated virtual cathode potential is computed as the value of $\Phi_{v c}$ for which the root of the polynomial equation in $G$ (Eq. 13) equals the value of $G$ given from Eq. 10 using the above expression $\gamma\left(\Phi_{f l}\right)$ for its 


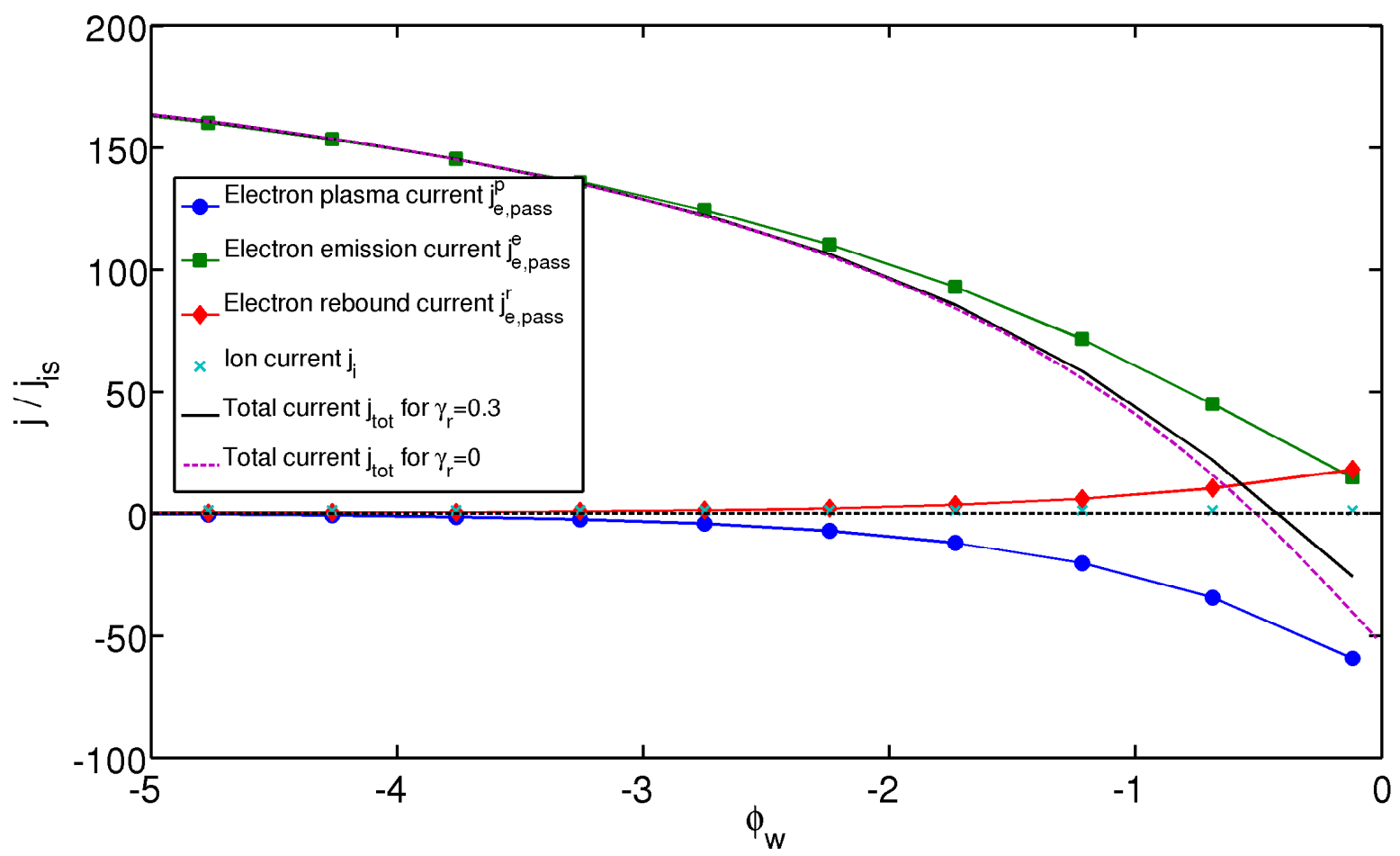

FIG. 3. Example of the different passing current densities flowing through an emissive surface under space-charge regime. The current densities are normalized to the ion saturation current density. The calculation was performed for an Argon plasma with $n_{0}=1 \times 10^{18} \mathrm{~m}^{-3} T_{e}^{p}=5 \mathrm{eV}, T_{e}^{e}=T_{w}=0.31 \mathrm{eV}, \gamma_{r}=0.3, \varphi_{\text {work }}=4.5 \mathrm{eV}$ and $A_{0}=1.2 \times 10^{6} \mathrm{Am}^{-2} \mathrm{~K}^{-2}$ (tungsten surface).

calculation. This is done numerically with a precision close to the computer precision and repeated for different sets of plasma parameters $\left(C, \gamma_{r}\right.$ and $\left.m_{i} / m_{e}\right)$. $C$ is varied from 1 to 100 by step of $1, \gamma_{r}$ from 0 to 0.8 by step of 0.01 and $m_{i} / m_{e}$ from 1836 to $100 \times 1836$ by step of $10 \times 1836$. We then proceed to a multivariable polynomial fit of the numerical $\Phi_{v c}$ to the seventh order of the form

$$
f\left(C, \gamma_{r}, \frac{m_{i}}{m_{e}}\right)=\sum_{n=0}^{7} \sum_{m=0}^{7} \sum_{p=0}^{7} a_{n, m, p} C^{n} \gamma_{r}^{m}\left(\frac{m_{i}}{m_{e}}\right)^{p}
$$

where $\left(a_{n, m, p}\right)_{(n, m, p) \in[0,7]^{3}}$ are polynomial coefficients.

This leads to an estimation of $\Phi_{v c}$ that can be used to compute the floating potential $\Phi_{f l}$ in Eq. 17 for a non zero electron emission temperature $T_{e}^{e}$, extanding the work of Hobbs and Wesson [9]. It is worth mentioning that the discrepancy between the numerical value of $\Phi_{v c}$ and the fit is always lower than $1 \%$ for $\gamma_{r} \leq 0.8$ (which covers any practical situation), and that the numerical procedure ensured that the computed total current density reaching the floating wall is lower than $1 \%$ of the ion saturation current $\left(j_{t o t} \sim 0\right)$. Numerical computations show that increasing the density $n_{0}$, the plasma electron temperature $T_{e}^{p}$ or decreasing the percentage of electrons that rebound on the wall $\gamma_{r}$ decrease the floating potential value for a given wall temperature. Consequently, the model predicts that it is more difficult to reach the plasma potential with a floating emissive material in tokamaks than in low temperature and density plasmas.

\section{CONFRONTATION OF THE MODEL WITH EXPERIMENTAL RESULTS (STATIC CASE)}

\section{A. Main experimental parameters}

The above theoretical model will now be compared with sets of experimental data from two experiments whose results were reported earlier. The first set of experiment was conducted in the Mirabelle device [4] and the two others 
on the von-Kármán plasma machine $[5,16]$ - these latter sets will be referred to as VKP1 and VKP2. The mean plasma parameters of these experiments are recalled in Tab. I. These parameters were obtained using electrostatic probes, consisting of a small tungsten loop wire of diameter $0.2 \mathrm{~mm}$ with $4 \mathrm{~mm}$ length in the Mirabelle's case and $8 \mathrm{~mm}$ in the VKP experiment, operated as cold Langmuir probes. In the remaining of this article, we report measurements using theses probes as emissive probes, i.e. when the tungsten loops are heated by Joule dissipation of a DC current to reach strong emission regime. The work function $\varphi_{\text {work }}$ for tungsten material is assumed to be 4.5 eV [7, 14] as a rough estimation, since it highly depends on surface contamination, crystal lattice and material deformation. We take the Dushman's constant $A_{0}$ to be equal to $1.2 \times 10^{6} \mathrm{Am}^{-2} \mathrm{~K}^{-2}$ for tungsten [7]. Note that comparing these experimental results with a 1D space-charge model can only be viewed as a rough comparison due to the geometry of the tungsten filaments. However, the present comparison demonstrate the basic features of the space-charge limited emission model and the validity of the hypothesis used.

\begin{tabular}{|c|c|c|c|c|c|c|}
\cline { 2 - 7 } \multicolumn{1}{c|}{} & gas & $T_{e}^{P}(\mathrm{eV})$ & $\varphi_{p}(\mathrm{~V})$ & $n_{0}\left(\times 10^{16} \mathrm{~m}^{-3}\right)$ & $\mathrm{B}(\mathrm{mT})$ & $\mathrm{P}(\mathrm{mbar})$ \\
\hline Mirabelle & $\mathrm{Ar}$ & 3.6 & 5 & {$[1$ to 10$]$} & 30 & $3 \times 10^{-4}$ \\
\hline VKP 1 & $\mathrm{Ar}$ & 4.6 & 7.1 & 12 & 5 & $4 \times 10^{-3}$ \\
\hline VKP 2 & $\mathrm{Ar}$ & 5.6 & 12.5 & 9.2 & 5 & $1 \times 10^{-3}$ \\
\hline
\end{tabular}

TABLE I. Main average parameters taken from an experiment performed on the linear plasma device Mirabelle [4] and two experiments performed on the von-Karman plasma experiment [5]. Notice that the mean density $n_{0}$ for the Mirabelle's case is a rough estimation as a current leak in the electronic was affecting the measurement. $B$ is the mean magnetic field at the probe position and $P$ the neutral pressure during the discharge.

\section{B. Space-charge criteria}

The transition between the emission current limited by thermoemission and space-charge is controlled by the amount of emitted electrons relatively to the primary electrons. For a given set of plasma and wall parameters (material, area), the control parameter is the wall emissivity, or equivalently the wall temperature $T_{w}$ when the emitted current follows the Richardson-Dushmann law. The onset for the development of the virtual cathode, as already mentioned in Ref. [24], is obtained when applying the above space-charge limited model for which the virtual cathode is located at the wall $\Phi_{w}=\Phi_{v c}$. Assuming that the temperature of emitted electrons is that of the wall $T_{e}^{e}=T_{w}$ (or in other words $C=C^{\prime}$ ), the threshold wall temperature $T_{w, t}$ is obtained using Eq. 17

$$
-\Phi_{\text {work }}+\frac{1}{C} \ln \left[\frac{A_{0} T_{w, t}^{2}}{e n_{0} M c_{s}} \sqrt{\frac{-m_{e}}{8 \Phi_{v c} m_{i}}} \frac{1+A G+A_{r} G \gamma_{r} / \gamma}{G}\right]=0
$$

This equation gives a criterion on $T_{w}$ for space-charge effect to occur depending on the plasma density $n_{0}$ and primary electron temperature $T_{e}^{p}$ (or on $j_{i s}$ in Eq. 17) and on the probe material emissivity (through $A_{0}$ and $\varphi_{\text {work }}$ ).

\section{Experimental evidence for a transition from thermoionic to space-charge regime}

In this subsection, experimental clues of a transition between thermoemission limited and space-charge limited regimes in the three experimental dataset mentioned above are analyzed applying the model developed in the previous section. It provides a theoretical estimate of the emissive probe temperature for this transition to occur. In Fig. 3 of [4] and from Fig. 5 and 3 of [5], the experimentally measured standard deviation of the floating potential of an emissive probe follows quite well the theoretical standard deviation as calculated from the model for temperature limited emission regime, up to a given emission current (or equivalently a given wall temperature). Then, the experimental points depart significantly from this model, meaning that the model assuming thermoemission limited current is no more valid. This transition is supposed to be related to a transition to space-charge limited emission. In particular, this is observed in Fig. 3 of Ref. [4], as the emission current $I_{e m}$ is greater than $1 \mathrm{~mA}$ and in Fig. 5 and 3 of Ref. [5], as $I_{e m} \sim 20 \mathrm{~mA}$ for the dataset VKP2 or less clearly as $I_{e m} \sim 30 \mathrm{~mA}$ for the dataset VKP1.

The key experimental parameters at the transition (i.e. the emission current $I_{\text {em }}^{t}$ and the corresponding probe potential $\left.\varphi_{w, t}\right)$ are reported in the first two columns of Tab. II. By inputting in Eq. 20) the experimental values given in Tab. I, the emitted electron temperature at the transition $T_{w, t}$ is estimated. We also assume here that $T_{w}=T_{e}^{e}$ (leading to $\left.C^{\prime}=C\right)$. The theoretical value of the wall potential at the transition is obtained as $\varphi_{w, t}=\varphi_{v c}\left(T_{w, t}\right)$ 
using the multivariable polynomial fit for calculating $\varphi_{v c}\left(T_{w, t}\right)$. These theoretical values are reported in the rightmost columns of Tab. II. The probe temperatures at the transition computed from the model lie between 2300 and $2500 \mathrm{~K}$ for the three experiments, which are reasonable values. The theoretical values of $\varphi_{w, t}$ lie in the transition interval observed experimentally. This is in agreement with the interpretation of the regime change observed experimentally and the transition towards space-charge limited regime, and with Eq. 17 holding for values of $I_{e m}$ above $I_{e m}^{t}$.

\begin{tabular}{|c|c|c|c|c|}
\cline { 2 - 5 } \multicolumn{1}{c|}{} & Exp. $I_{e m}^{t}$ & Exp. $\varphi_{w, t}$ & Th. $T_{w, t}$ & Th. $\varphi_{w, t}$ \\
\hline data Mirabelle & $0.9-1.1 \mathrm{~mA}$ & $1.2-1.9 \mathrm{~V}$ & $2340 \mathrm{~K}$ & $2.2 \mathrm{~V}$ \\
\hline data 1 VKP & $30-35 \mathrm{~mA}$ & $3.5-4.1 \mathrm{~V}$ & $2450 \mathrm{~K}$ & $3.5 \mathrm{~V}$ \\
\hline data 2 VKP & $15-20 \mathrm{~mA}$ & $7.1-8.6 \mathrm{~V}$ & $2420 \mathrm{~K}$ & $8.1 \mathrm{~V}$ \\
\hline
\end{tabular}

TABLE II. Transition parameters $I_{e m}^{t}$ and $\Phi_{w}, t$ estimated from the data of Refs. [4, 5] and theoretical values estimated using the criteria of Eq. 20 assuming $T_{w}=T_{e}^{e}$. The density in the Mirabelle's case is taken to be $5 \times 10^{16} \mathrm{~m}^{-3}$. We assume the parameter $\gamma_{r}$ to be equal to 0.3 .

\section{Average emissive probe potential in the space-charge limited regime}

For heating currents above $I_{e m}^{t}$ (i.e. in the space-charge current limited regime), the floating potential of the probe of the aforementioned experiments is predicted using the polynomial fit of $\Phi_{v c}$ (Eq. 19) in Eq. 17 . However this prediction requires the knowledge of $T_{w}$ and $T_{e}^{e}$. Since none of these parameters were experimentally accessible the following procedure is adopted: we first assume $T_{w}=T_{e}^{e}$ and then the evolution of the floating probe potential is computed for two values of $T_{w}$, namely at the onset of the space-charge regime $\left(T_{w} \sim 2550 \mathrm{~K}=0.22 \mathrm{eV}\right)$, and tungsten melting point $\left(T_{w} \sim 3700 \mathrm{~K}=0.32 \mathrm{eV}\right)$. The evolution of the predicted probe floating potential value as a function of $\gamma_{r}$ is shown in Fig. 4 for the two aforementionned temperature $T_{w}$. Together with these predicted theoretical values, Fig. 4 displays the plasma potential value $\varphi_{p}$ measured from analysis of cold Langmuir probes $I$ - $V$ characteristics as well as the average values of the probe floating potential $\varphi_{f l, \text { max }}$ measured from emissive probes, and that correspond to the maximum values of the emission current $I_{e m}$ achieved in the three experiments.

For the three cases under investigation, the experimental probe floating potentials lie within the range of floating potential predicted from theory (which indeed strongly depends on $T_{w}$ and $\gamma_{r}$ ). In the Mirabelle's case, the maximum floating potential achieved experimentally lies in between the two circled blue and squared red curves even for a zero $\gamma_{r}=0$ (no rebound electrons). However, even for the highest probe temperature $\left(T_{w}=0.32 \mathrm{eV}\right.$ at the melting point), the model cannot explain the VKP experimental observations if no rebound electrons are considered. This clearly shows the importance of taking into account rebound electrons in the derivation of the model. In addition, note that the higher the plasma temperature (graphs from left to right in Fig. 4), the hotter the emissive probe should be in order to reach the plasma potential, and that the probe would probably be melting/breaking before reaching it in the two VKP experiment cases.

\section{INFLUENCE OF PLASMA PARAMETER FLUCTUATIONS ON FLOATING EMISSIVE PROBE FLUCTUATIONS IN THE SPACE-CHARGE LIMITED REGIME}

The floating potential of an emissive probe emitting enough electrons to float near the plasma potential mean value is usually believed to measure fluctuations of the plasma potential. However, it has recently been demonstrated in Ref. [4] and [5] that plasma density and electron temperature fluctuations also play a role on the floating potential of the probe. In the two aforementionned articles, it has been explained theoretically why it is so when the emission current is limited by thermoemission but not when the probe is under space-charge regime. In this section, the sheath model in the space-charge current limited regime derived in section II is applied to the experimental data to see the influence of both electron density and temperature fluctuations using on the probe potential. The influence of the rebound electrons on the dynamics of the fluctuations of the floating potential is also discussed.

\section{A. First order expansion from the model}

The model presented in section II was derived for static plasma parameters. However, as emissive probe measurements are usually used to probe low frequency fluctuations of the plasma potential, we use the model to account 

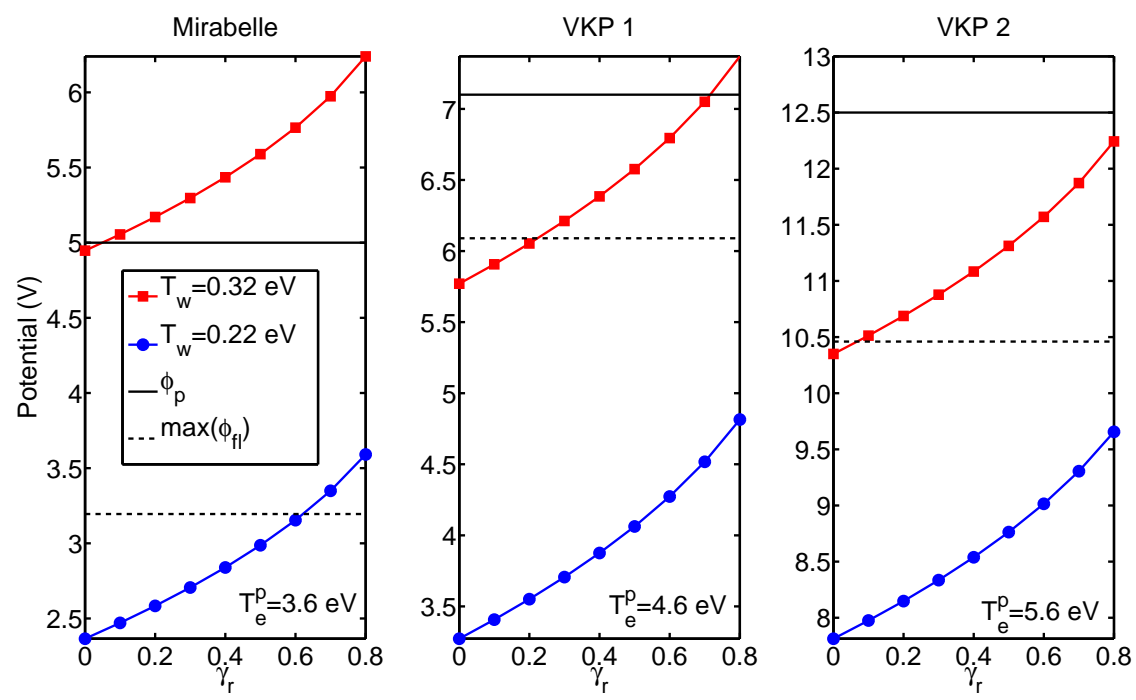

FIG. 4. Average floating potential obtained with Eq. 17 assuming $T_{w}=T_{e}^{e}$ and using data from Tab. I. Left: Mirabelle experiment, center: VKP 1, right: VKP 2. The floating potential $\varphi_{f l, m a x}$ corresponds to the maximum of the probe floating potential obtained experimentally. The plasma potential $\varphi_{p}$ was obtained from cold probe analysis.

for the effect of slow plasma parameter fluctuations (i.e. fluctuations whose frequency is much smaller than the ion plasma frequency). To get an insight into the influence of the fluctuations of $T_{e}^{p}$ and $n_{0}$ on the probe, a first order expansion of Eq. 17 is done. In the following the wall temperature $T_{w}$ is assumed to be constant as thermal frequency responses of the material are much slower than plasma fluctuation frequencies of interest (in the kiloHertz range) and $T_{e}^{e}=T_{w}$ is once again assumed. A first order expansion of the floating potential reads:

$$
\Delta \varphi_{f l}=\frac{\partial \varphi_{f l}}{\partial \varphi_{p}} \Delta \varphi_{p}+\frac{\partial \varphi_{f l}}{\partial \bar{n}} \Delta \bar{n}+\frac{\partial \varphi_{f l}}{\partial T_{e}^{p}} \Delta T_{e}^{p} \Rightarrow \Delta \bar{\varphi}_{f l}=\frac{\Delta \varphi_{f l}}{\left\langle T_{e}^{p}\right\rangle}=\frac{\partial \varphi_{f l}}{\partial \varphi_{p}} \Delta \bar{\varphi}_{p}+\frac{\partial \bar{\varphi}_{f l}}{\partial \bar{n}} \Delta \bar{n}+\frac{\partial \varphi_{f l}}{\partial T_{e}^{p}} \Delta \bar{T}_{e}^{p}
$$

where $\bar{f}$ means normalized quantities (the density is normalized to $\left\langle n_{0}\right\rangle$, while potentials and electron temperature are normalized to $\left.\left\langle T_{e}^{p}\right\rangle\right)$ and \langle\rangle stands for mean value. The relative amplitudes of $\partial \bar{\varphi}_{f l} / \partial \bar{n}$ and $\partial \varphi_{f l} / \partial T_{e}^{p}$ are compared to $\partial \varphi_{f l} / \partial \varphi_{p}=1$ in the following two subsections.

Plasma density fluctuations The dependence of $\bar{\varphi}_{f l}$ on the plasma density is readily estimated as $\bar{\varphi}_{v c}$ depends only on $C=T_{e}^{p} / T_{w}\left(m_{i} / m_{e}\right.$ is a constant and $\gamma_{r}$ is supposed to remain constant during one given experiment). The plasma density then only appears in $j_{i s}$ (Eq. 17). Doing a first order Taylor expansion by setting $n_{0}=\left\langle n_{0}\right\rangle+\delta n_{0}$ with $\delta n_{0} \ll\left\langle n_{0}\right\rangle$, one get $\partial \bar{\varphi}_{f l} / \partial \bar{n}=-1 /\langle C\rangle$. In low temperature plasmas $\langle C\rangle=\left\langle T_{e}^{p}\right\rangle / T_{w} \in[6-20]$ which leads to a discrepancy of about $5-15 \%$ in the measurement of the plasma potential fluctuations. Note that in tokamak $\langle C\rangle \gg 100$ so that according to our 1D model, density fluctuations do not affect emissive probe measurements in the space-charge limited regime.

Plasma temperature fluctuations Plasma temperature fluctuations influence on the floating potential of the probe can only be computed numerically as the dependence of $\Phi_{v c}$ upon $C$ is non-trivial. Looking at Eq. 17, one can notice that five parameters affect $\Phi_{f l}: T_{e}^{p}, T_{w}, \gamma_{r}, m_{i} / m_{e}, n_{0}$ as $A_{0}$ is set to $1.2 \times 10^{6} \mathrm{Am}^{-2} \mathrm{~K}^{-2}$ for tungsten wall. $\partial \varphi_{f l} / \partial T_{e}^{p}$ is then computed for different set of parameters, changing one parameter at a time and for a wide range of mean plasma temperature value $T_{e}^{p}$. The results are as follow.

First, $\partial \varphi_{f l} / \partial T_{e}^{p}$ hardly depends on the wall temperature $T_{w}$ (or equivalently $C$ since we assume $T_{w}=T_{e}^{e}$ ) nor on the plasma density $\left\langle n_{0}\right\rangle$. This has been tested by changing $T_{w}$ from $0.22 \mathrm{eV}$ (onset value for space-charge regime to occur) to its highest value for tungsten $(0.32 \mathrm{eV})$ and by increasing $\left\langle n_{0}\right\rangle$ by three orders of magnitude (from $10^{17} \mathrm{~m}^{-3}$ up to $10^{20} \mathrm{~m}^{-3}$ to reach tokamak values). In all cases, $\partial \varphi_{f l} / \partial T_{e}^{p}$ remains quite unchanged, so we conclude that both parameters $T_{w}$ and $n_{0}$ have a very weak influence on floating potential fluctuations.

The effect of the gas mass is rather weak. From an Argon (like Mirabelle and VKP discharges presented here) to a Hydrogren plasma (in tokamak for instance), the influence of temperature fluctuations on $\varphi_{f l}$ would only be diminished by less than $5 \%$. However, changing the rebound coefficient $\gamma_{r}$ has a much stronger effect as one can see in Fig. 5. Increasing the value of $\gamma_{r}$ from 0 to 0.7 decreases the influence of temperature fluctuations by about 


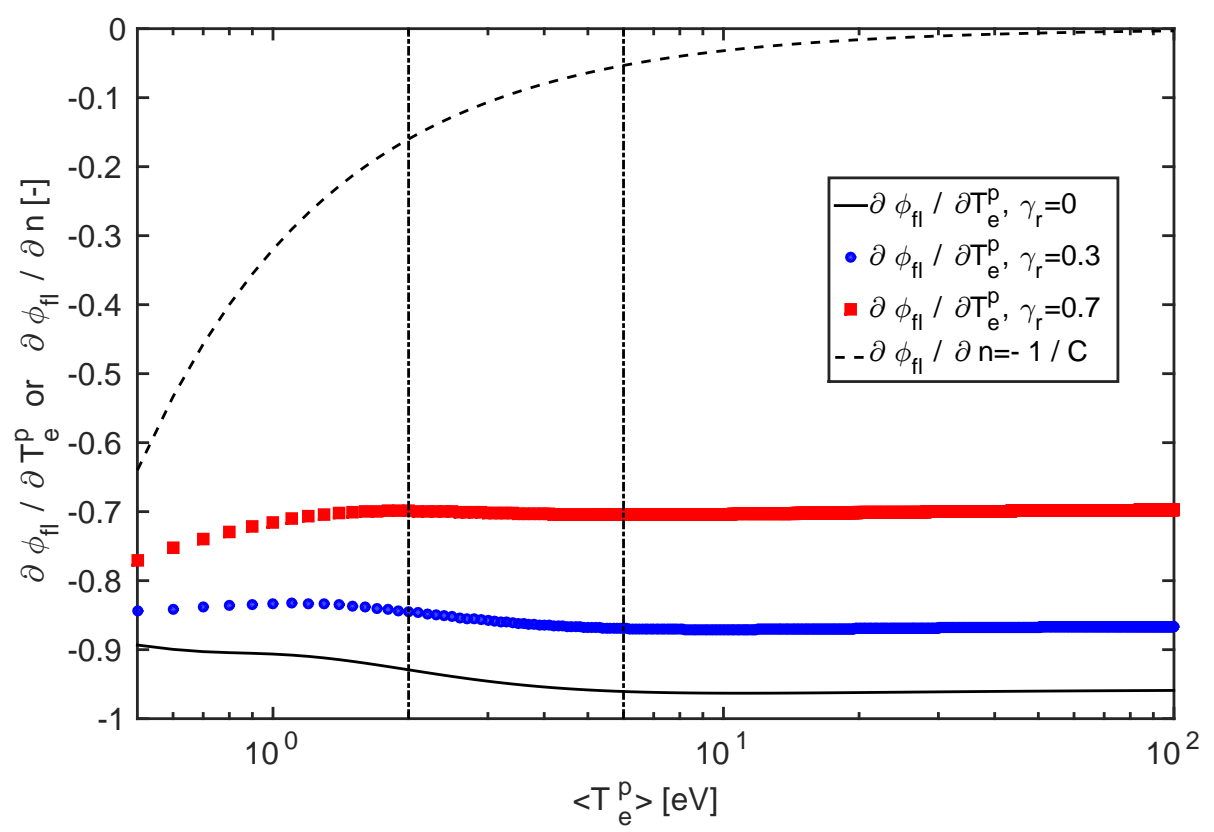

FIG. 5. Variation of the partial derivative of $\varphi_{f l}$ with respect to the plasma temperature $T_{e}^{p}$ as a function of $<T_{e}^{p}>$. Each curves were calculated by numerically differentiating Eq. 17. The black solid line is obtained for an Argon gas, $<n_{0}>=1 \times 10^{17}$ $\mathrm{m}^{-3}, \gamma_{r}=0, T_{w}=T_{e}^{e}=0.25 \mathrm{eV}$ and $A_{0}=1.2 \times 10^{6} \mathrm{Am}^{-2} \mathrm{~K}^{-2}$. The circled blue and the squared red curves are plotted for the same parameters except for $\gamma_{r}$ that is changed to 0.3 and 0.7 , respectively. For comparison, we also display the partial derivative of $\bar{\varphi}_{f l}$ with respect to the normalized density $\bar{n}$ (the black dashed line, $-1 / \mathrm{C}$ ) for $<T_{e}^{e}>=0.32 \mathrm{eV}$ as well as the $\left\langle T_{e}^{p}\right\rangle$ boundaries corresponding to low temperature plasmas (2-6 eV - vertical dashed lines).

$25 \%$ when $T_{e}^{p}$ is high enough. For a reasonable value of $\gamma_{r}$, i.e. 0.3 , the fluctuations would be diminished by more than $10 \%$ compare to $\gamma_{r}=0$. The fact that the influence of temperature fluctuations diminishes with $\gamma_{r}$ can be easily understood as increasing $\gamma_{r}$ results in replacing slow emission electrons by fast rebound electrons whose current compensates exactly a fraction of the current of primary electrons that fluctuates with $\left\langle T_{e}^{p}\right\rangle$.

Then, one can notice that $\partial \varphi_{f l} / \partial T_{e}^{p}$ for all the curves in Fig. 5 quickly saturates to some constant value by increasing $\left\langle T_{e}^{p}>\right.$, meaning that the fluctuations of temperature affects $\varphi_{f l}$ in low, as well as in high, temperature plasmas. Last but not least, temperature fluctuations have a much stronger influence on $\bar{\varphi}_{f l}$ than density ones as $\partial \varphi_{f l} / \partial T_{e}^{p}$ is much greater (in absolute value) than $\partial \bar{\varphi}_{f l} / \partial \bar{n}=-1 /\langle C\rangle$ shown by a dashed line in Fig 5 . The contribution of temperature fluctuations on the floating potential are actually quite significant with respect to the ones of the plasma potential fluctuations (a bit lower in absolute value). This means that $\varphi_{f l}$ fluctuations are strongly affected by $T_{e}^{p}$ fluctuations and thus that the floating potential fluctuations of an emissive probe differs from the ones of the plasma potential according to the model, eventhough reduced by taking into account $\gamma_{r}$.

\section{B. Comparison to experimental data: VKP data}

In this subsection, the experimental floating potential fluctuations measured by an emissive probe under spacecharge limited current regime are compared to the fluctuations given by the model derivated in section II (Eq. 17), the experimental fluctuations of the plasma parameters $\left(T_{e}^{p}, n_{e}\right.$ and $\left.\varphi_{p}\right)$ being the inputs of the model. Data obtained on Mirabelle could not be processed since the time evolution of the temperature was not measured and density fluctuations measurements were not precise enough. We thus focus on the VKP experiment [5] for which, a conditional reconstruction method of the $I-V$ characteristics [17] has been used, enabling the measurements of all the interesting time series $\left(T_{e}^{p}, n_{e}, \varphi_{f l}, \varphi_{p}\right.$ and $\left.\varphi_{f l, e m}\right)$. The only drawback of this experiment could lie in the way the plasma is created in the VKP device: using radiofrequency coupling. Thus, the electron temperature could be over estimated and there could be errors on its phase measurements - although previous measurements with compensated cold Langmuir probes $[6,22]$ did not show significant differences with measurements with uncompensated cold Langmuir probes. The time series used here are those shown in Fig. 4 of [5], corresponding to emission regimes when the mean floating potential was the closest to the plasma potential (i.e. for the largest emission current). In 


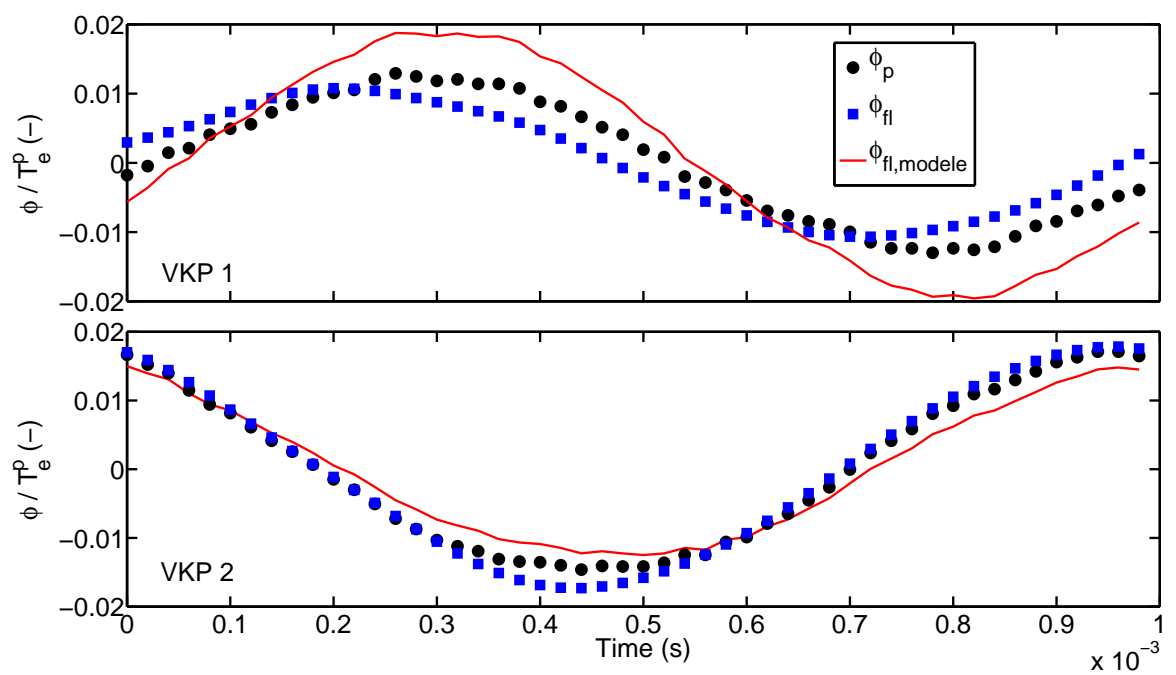

FIG. 6. Fluctuations of the normalized plasma potential (black dots), experimental floating potential (blue squares) and theoretical floating potential (red solid lines) as a function of time for a) VKP1 and b) VKP2. The theoretical red curves are calculated using the experimental time series plugged in Eq. 17 and assuming $T_{w}=T_{e}^{e}$. In a) and b), we used $T_{w}=0.31 \mathrm{eV}$ and $\gamma_{r}=0.3$.

\begin{tabular}{|c|c|c|}
\hline & VKP 1 & VKP 2 \\
\hline \hline Experiment & $0.035 \mathrm{~V}$ & $0.069 \mathrm{~V}$ \\
\hline Model $\gamma_{r}=0$ & $0.065 \mathrm{~V}$ & $0.053 \mathrm{~V}$ \\
\hline Model $\gamma_{r}=0.3$ & $0.062 \mathrm{~V}$ & $0.054 \mathrm{~V}$ \\
\hline Model $\gamma_{r}=0.7$ & $0.058 \mathrm{~V}$ & $0.056 \mathrm{~V}$ \\
\hline
\end{tabular}

TABLE III. Experimental and theoretical standard deviations of the floating potential of the emissive probe for the two VKP experiments. The theoretical ones are calculated using the experimental time series plugged in Eq. 17 and setting $T_{w}=T_{e}^{e}$ to $0.31 \mathrm{eV}$.

both cases, according to results shown in section III C of this article, the current is obviously space-charge limited. The experimental fluctuations of the floating potential obtained in both regimes are shown as blue square symbols in both panels of Fig. 6. The associated plasma potential fluctuations are displayed as black circles. In order to compare these time-series to the space-charge model, $T_{w}=T_{e}^{e}$ is assumed and $\gamma_{r}$ is set to a realistic value of $0.3 . T_{w}$ is computed to ensure equality between the mean potential found using the space-charge model and the experimental data, leading to $T_{w}=0.31 \mathrm{eV}$ for both experiments. The theoretical curves correspond to the red solid curves in Fig. 6 .

In the first experiment, the standard deviation given by the model is $0.062 \mathrm{~V}$ while experimentally it is about $0.035 \mathrm{~V}$, and in the second, the modeled standard deviation is $0.054 \mathrm{~V}$ for an experimental one of $0.069 \mathrm{~V}$ (see Tab. III). This discrepancy between the model and the experimental data cannot be explained by the choice of the a priori value we took for $T_{w}$ and $\gamma_{r}$. We have shown in Sec. IV A that changing $T_{w}$ does not affect much the standard deviation, in agreement with the experimental data under space-charge regime for which changing $I_{e m}$, so $T_{w}$, does not affect much the standard deviation (see Fig. 3 and 5 of Ref. [5]). However, we shown in the same section that changing $\gamma_{r}$ affects the floating potential fluctuations. In Tab. III, it can be seen that changing $\gamma_{r}$ from 0 to 0.7 affects the modeled standard deviation of about $11 \%$ in VKP 1 and $5 \%$ in VKP 2. Eventhough significant, the rebound electrons does not suffice to explain the discrepancy between the model and the experimental data. More experimental verification (where the 1D assumption would be more accurate to match the model) or theoretical developments including more complex geometries should be investigated to draw any clear conclusion. Nevertheless, rebound electrons would certainly be an important ingredient as they replace part of the emitted current by a current fluctuating like the electron plasma current.

Even if the experimental value of the floating potential fluctuations cannot be obtained when varying $\gamma_{r}$, the two sets of experiments described in this subsection provide a first experimental clue that taking into account rebound electrons is a key parameter in the interpretation of the floating potential fluctuations of emissive probes. Indeed, 
as shown above, the value of $\gamma_{r}$ controls the influence of electron temperature fluctuations on the floating potential fluctuations. Table III demonstrates that modifications of $\gamma_{r}$ may increase of decrease the amplitude of the fluctuations of the floating potential. Although the set of experiments shown in the present article does not provide a definitive experimental validation of the space-charge current limited model taking into account rebound electrons, it clearly shows that a correct and detailed analysis of the fluctuations of emissive probes requires to consider these electrons. These sets of experimental data also support the need for other experimental verification (where the 1D assumption would be more accurate) or theoretical developments including more complex geometries.

\section{CONCLUSIONS AND DISCUSSIONS}

In this article, an improved version of the 1D model developed by Takamura [23] is presented. Namely, the modeling of the total current flowing through an emissive probe under space-charge regime is modified by taking into account secondary electron emission due to the retrodiffusion of primary electrons on the hot surface. We then proceed to obtain a relation linking the probe potential to the virtual cathode potential. From this relation, we derive a criterion on the wall temperature with respect to the plasma and wall parameters for the transition between thermoemission to space-charge regimes to occur. Last, in the peculiar case of the floating potential, a numerical estimation of the virtual cathode potential is obtained for a large range of experimental parameters. This allows to apply the model to experimental floating potential data from two different experiments.

We first show in section III the importance of taking into account rebound electrons in the model derivation to reproduce the mean floating potential of the experimental observations. We also show that the transition between the thermoemission regime to the space-charge one agrees with the previous observations [4,5] and appears for probe temperatures around 2300-2500 K. More systematic experiments could be done to verify this last point by measuring experimentally the probe temperature. In addition, tests with different probe materials, thus changing the work function and the wall temperature transition value, could also provide useful information.

Then, we study the effect of low frequency plasma parameter fluctuations (compared to the plasma ion frequency) on the floating potential of an emissive probe given by the static space-charge model. The model shows that plasma density fluctuations have a very weak effect for low temperature plasmas (2-5 eV) and are completely negligible for high temperature plasmas $(>5 \mathrm{eV}$, tokamaks). Thus plasma density fluctuations hardly contribute to floating potential fluctuations. Plasma temperature fluctuations, however, have a non-negligible effect on the probe floating potential meaning that inferring plasma potential fluctuations from emissive probes would not be straightforward. Nevertheless, according to this model, taking into account rebound electrons results into a lower influence of plasma electron temperature fluctuations, whose influence decreases with $\gamma_{r}$.

We then proceed to compare the 1D model with the experimental low floating potential fluctuations of an emissive probe under space-charge regime. Unfortunately, the theory does not properly model fluctuations of the floating potential. To gain further insight on the model validity, the experimental results using controlled driving of harmonic fluctuations presented here should be extended in the future to situations where the time evolution of the electron temperature is probed with a high accuracy. Moreover, the $1 \mathrm{D}$ theoretical model was applied to experiments were $2 \mathrm{D}$ effects are unavoidable (potential gradient along the tungsten filament) [15]. In a foreseen work, plane surface LaB6 probe heated by laser, as suggested by Schrittwieser et al. [19], should be used. In that case, the mean potential on the probe surface is homogeneous, as no strong DC current is circulating in the probe.

\section{ACKNOWLEDGEMENT}

This work has been partly funded by the French National Research Agency under the two contracts ANR-13-JS040003-01 and ANR-11-BS09-023-03 Sediba.

[1] Fusion research in austria - activities report in 2014 and 2015.

[2] J. Adamek, J. Horacek, J. Seidl, H.W. Muller, R. Schrittwieser, F. Mehlmann, P. Vondracek, S. Ptak, COMPASS Team, and ASDEX Upgrade Team. Direct plasma potential measurements by ball-pen probe and self-emitting langmuir probe on compass and asdex upgrade. Contribution to Plasma Physics, 54(3):279-284, 2014.

[3] G. Bousselin, J. Cavalier, J. F. Pautex, S. Heuraux, N. Lemoine, and G. Bonhomme. Design and validation of the ball-pen probe for measurements in a low-temperature magnetized plasma. Review of Scientific Instruments, 84:013505 (1-8), 2013.

[4] G. Bousselin, N. Lemoine, J. Cavalier, S. Heuraux, and G. Bonhomme. On the measurement of plasma potential fluctuations using emissive probes. Review of Scientific Instruments, 85:056102(1-3), 2014. 
[5] G. Bousselin, N. Plihon, N. Lemoine, J. Cavalier, and S. Heuraux. How plasma parameters fluctuations influence emissive probe measurements. Physics of Plasmas, 22:053511(1-8), 2015.

[6] A. Cantin and R. Gagne. . Applied Physics Letters, 30:31, 1977.

[7] V. S. Fomenko. Handbook of thermoionic properties: electronic work functions and Richardson constants of elements and compounds. Plenum press data division, 1966.

[8] J. P. Gunn. Evidence for strong secondary electron emission in the tokamak scrape-off layer. Plasma Physics and Controlled Fusion, 54:085007 (7pp), 2012.

[9] G. D. Hobbs and J. A. Wesson. Heat flow through a langmuir sheath in the presence of electron emission. Plasma Physics, 9:85-87, 1966.

[10] R. F. Kemp and J. M. Sellen. . Review of Scientific Instruments, 37:455, 1966.

[11] I. H. Khan, J. P. Hobson, and R. A. Armstrong. Reflection and diffraction of slow electrons from single crystals of tungsten. Physical Review, 129(4):1513-1523, 1963.

[12] I Langmuir. . Phys. Rev., 21:419, 1922.

[13] W Li, J. X. Ma, J. Li, Y. Zheng, and M. Tan. . Physics of Plasmas, 19:030704, 2012.

[14] H. B. Michaelson. The work function of the elements and its periodicity. Journal of Applied Physics, 48(11):4729-4733, 1977.

[15] E. Mravlag and P. Krumm. Space potential measurements with a continuously emitting probe. Review of Scientific Instruments, 61:2164, 1990.

[16] N. Plihon, G. Bousselin, F.Palermo, J. Morales, W.J.T. Bos, F. Godeferd, M. Bourgoin, J.-F. Pinton, M. Moulin, and A. Aanesland. Flow dynamics and magnetic induction in the von-karman plasma experiment. Journal of Plasma Physics, 81:345810102 (1-17), 2015.

[17] N. Plihon, C. S. Corr, P. Chabert, and J. L. Raimbault. Periodic formation and propagation of double layers in the expanding chamber of an inductive discharge operating in Ar/SF 6 mixtures. Journal of Applied Physics, 98(2):1-7, 2005.

[18] B.S. Schneider, S. Costea, C. Ionita, R. Schrittwieser, V. Naulin, J. J. Rasmussen, R. Starz, N. Vianello, J. Kovacic, and T. Gyergyek. Robust highly emissive probe for plasma potential measurements in the edge region of toroidal plasmas. Proceedings of Science, 2015.

[19] R. Schrittwieser, C. Ionita, P. Balan, R. Gstrein, O. Grulke, T. Windisch, C. Brandt, T. Klinger, R. Madani, G. Amarandei, and A. K. Sarma. Laser-heated emissive plasma probe. Review of Scientific Instruments, 79:083508 (1-9), 2008.

[20] J P Sheehan and N Hershkowitz. Emissive probes. Plasma Sources Science and Technology, 20(6):063001, 2011.

[21] A. Siebenforcher and R. Schrittwieser. A new simple emissive probe. Review of Scientific Instruments, $67(849), 1996$.

[22] I D Sudit and F F Chen. Rf compensated probes for high-density discharges. Plasma Sources Science and Technology, 3(2):162, 1994.

[23] S. Takamura, N. Ohno, M.Y. Ye, and T. Kuwabara. Space-charge limited current from plasma-facing material surface. Contribution to Plasma Physics, 44(1-3):126-137, 2004.

[24] M. Y. Ye and S. Takamura. Effect of space-charge limited emission on measurements of plasma potential using emissive probes. Physics of Plasmas, 7:3457-3463, 2000. 